\title{
Vasovagal cardiac arrest during spinal anesthesia for Cesarean section -A case report-
}

\author{
Young-Eun Jang, Sang-Hwan Do, and In-Ae Song \\ Department of Anesthesiology and Pain Medicine, Seoul National University Bundang Hospital, Seongnam, Korea
}

The vasovagal response is characterized by an inappropriate combination of bradycardia and paradoxical vasodilation. During a general or neuraxial anesthesia-induced sympathectomy, a sudden vagal activation and/or an acute reduction in sympathetic tone can cause serious vasovagal responses. Neuraxial anesthesia for Cesarean section may trigger vasovagal response, due to multiple risk factors; high neuraxial block, sudden hemorrhage, aortocarval compression, peritoneal manipulation, and emotional stress. A 39-year-old pregnant woman, at 38 weeks of gestation with episodes of non-sustained ventricular arrhythmia and newly developed vasovagal syncope during pregnancy, was scheduled to undergo a spinal anesthesia for an elective Cesarean section. Immediately after the placental expulsion, a sudden severe bradycardia, followed by a cardiac arrest occurred. The patient fully recovered after prompt cardiopulmonary resuscitation with chest compression, manual ventilation with oxygen, rapid injection of epinephrine and hydration. This case illustrates a serious potential risk of vasovagal response superimposed on neuraxial anesthesia, during a Cesarean section, especially during placental expulsion. (Korean J Anesthesiol 2013; 64: 77-81)

Key Words: Anesthesia, Cesarean section, Heart arrest, Spinal, Syncope, Vasovagal.

Vasovagal response (neurocardiogenic response) is the most common mechanism of syncope. It is characterized by an inappropriate combination of bradycardia and paradoxical vasodilation. A variety of mechanisms can cause vasovagal response [1].

Central triggers, such as severe pain, anxiety, fear, and emotional stress, can cause vasovagal response. Syncope and hemodynamic collapses, due to pain, anxiety and fear during medical procedures, such as vascular access or induction of neuraxial anesthesia, are good examples [2-4]. Usually, patients' past medical history reveals previous vasovagal episodes and susceptibility.

Sudden parasympathetic activation (also known as vagotonia) and sympathetic withdrawal can cause vasovagal

Received: February 29, 2012. Revised: 1st, March 29, 2012; 2nd, April 19, 2012. Accepted: April $26,2012$.

Corresponding author: In-Ae Song, M.D., Department of Anesthesiology and Pain Medicine, Seoul National University Bundang Hospital, 166, Gumi-ro, Bundang-gu, Seongnam 463-707, Korea. Tel: 82-31-787-7499, Fax: 82-31-787-4063, E-mail: nodame1@naver.com

C) This is an open-access article distributed under the terms of the Creative Commons Attribution Non-Commercial License (http:// creativecommons.org/licenses/by-nc/3.0/), which permits unrestricted non-commercial use, distribution, and reproduction in any medium, provided the original work is properly cited. 
response. Mechanical and chemical stimuli from the collapsed left ventricular wall can activate afferent fibers to the brainstem, which can stimulate parasympathetic activity and inhibit sympathetic activity to cause a bradycardia, and vasodepressor response: the classic Bezold-Jarish reflex. Sudden decrease of venous return can activate such pathway [5-7]: high neuraxial anesthesia, postural change, massive hemorrhage, systemic vasodilation, and special conditions, such as aortocarval compression in full-term women. Also reflex bradycardia is a well-known cause of a severe vagal activation [8]. Along with the oculocardiac reflex and the laryngeal reflex, surgical manipulations of the abdominal contents, peritoneum or traction of the visceral ligaments often cause a severe bradycardia and hypotension, during an operation [9].

In daily life, most vasovagal episodes are self-limited, and are easily treated. However, the sympathetic compensatory mechanism is usually blocked, during general anesthesia or neuraxial block-induced sympathectomy. Therefore, a patient is more susceptible to vasovagal response. Thus, when many risk factors are combined, serious outcomes, such as cardiovascular collapse, shock or cardiac arrest can occur $[10,11]$.

\section{Case Report}

A 39-year-old woman, (gravida 7, para 1, [weight $67 \mathrm{~kg}$, height $152 \mathrm{~cm}$ ]) at 38 weeks of gestation, was admitted for an elective Cesarean section. She had a history of self-limited palpitation, about 3-5 times per day. This palpitation began approximately 10 years ago and usually stopped within a few seconds, without other significant symptoms. During pregnancy, she had experienced new symptoms: episodes of sudden nausea, general weakness, and dizziness after prolonged standing.

These symptoms were easily relieved by sitting or squatting. The preoperative blood test, urinalysis, chest X-ray, electrocardiogram (ECG), and echocardiogram were all normal. However, her 24 hour Holter monitoring revealed one episode of non-sustained ventricular tachycardia (NSVT). Five to six hours before the surgery, she felt the same self-limited palpitation, but no other symptoms were observed.

The patient arrived at the operating room without premedication. We started our routine monitoring with ECG, noninvasive arterial pressure, and oxygen saturation. Supplemental oxygen was administrated by a facial mask with a reservoir bag at the rate of $6 \mathrm{~L} / \mathrm{min}$. Her vital signs were a heart rate (HR) of 92-95 bpm with normal sinus rhythm, 103-104/63-64 mmHg of blood pressure (BP), and $100 \%$ of oxygen saturation $\left(\mathrm{SpO}_{2}\right)$. Because she had a history of NSVT, a 20-gauge catheter was inserted, at her left radial artery, for continuous arterial pressure monitoring. After positioning the patient to the right lateral decubitus, $8 \mathrm{mg}$ of $0.5 \%$ hyperbaric bupivacaine, mixed with $20 \mu \mathrm{g}$ of fentanyl, was injected with a 25 -gauge spinal needle at L4-5 interspace. Her vital signs were $92 \mathrm{bpm}$ of $\mathrm{HR}, 102 / 63 \mathrm{mmHg}$ of $\mathrm{BP}$ and $100 \%$ of $\mathrm{SpO}_{2}$, immediately after spinal anesthesia. Then, the patient was put in a supine position with a left lateral tilt, and was given $50 \mu \mathrm{g}$ of prophylactic phenylephrine intravenously for the prevention of hypotension after the spinal anesthesia. The initial block level, 2 minutes after the introduction of spinal anesthesia, was at T10 checked with cold sense using an alcohol swab. As the block height went higher, the patient showed hypotension of 88/40 mmHg with $95 \mathrm{bpm}$ of HR, and complained of nausea. Thus far, $700 \mathrm{ml}$ of Ringer's Lactated solution had been administrated intravenously. Relative hypovolemia, caused by alpha-blocking effect of spinal anesthesia, was the suspected cause of hypotension. Therefore, we immediately injected phenylephrine twice (50 $\mu$ g each), and continued hydration with hydroxyethyl starch (Voluven ${ }^{\circledR}$, Fresenius Kabi, Germany). Her vital signs were stabilized with $96 \mathrm{bpm}$ of HR and 140/74 mmHg of BP, without showing arrhythmia or reflex bradycardia, after phenylephrine administration, as well as her nausea stopped.

Sensory level to alcohol swab to the T3 was noticed, 10 minutes after the spinal anesthesia. Within 10 minutes of the skin incision, a $3.9 \mathrm{~kg}$ female was delivered with Apgar scores of 9 at both 1 and 5 minutes. The patient was not anxious or irritable, and showed stable vital signs. Her vital signs were $98 \mathrm{bpm}$ of HR with normal sinus rhythm, 128/68 mmHg of $\mathrm{BP}$, and $100 \%$ of $\mathrm{SpO}_{2}$. One gram of Cefazolin $\left(\right.$ Cefamezin ${ }^{\circledR}$, DONG-A Pharm, Korea), mixed with $10 \mathrm{ml}$ of normal saline, was administrated intravenously after a confirmation of a negative antibiotic skin test result. We suggested sedation with intravenous midazolam for the rest of the operation. However, she said she was alright and refused sedation.

Separation of the placenta from the uterus was not easy. Hence, a forced traction was done, repeatedly. Immediately after the placental expulsion, she suddenly looked pale and complained of severe nausea. Then she suddenly deteriorated with cardiovascular collapse, respiratory arrest, and loss of consciousness. The HR was decreased, precipitously. The QRS complex disappeared with only P-waves being observed; eventually asystole occurred. Cardio-pulmonary resuscitation (CPR) was started immediately with external cardiac compression, manual ventilation with $100 \%$ oxygen with ventilator mask, rapid hydration, and Trendelenburg position. After the injection of $1 \mathrm{mg}$ of epinephrine, along with 1 minute of CPR, the ECG showed narrow QRS complexes with irregular R-R interval. However, her systolic pressure remained at 30-40 $\mathrm{mmHg}$, without external cardiac compression. Approximately 3 minutes after CPR, the HR recovered up to $80-90 \mathrm{bpm}$ with 
regular narrow QRS complex and an effective arterial pressure (80-90/70-80 mmHg). The patient gradually recovered her consciousness, spontaneous breathing, and orientation within a few minutes, and was placed horizontally with caution. Ephedrine $10 \mathrm{mg}$ was administrated twice, when the systolic pressure was lower than $80 \mathrm{mmHg}$, followed by continuous infusion of dopamine at the rate of $5 \mu \mathrm{g} / \mathrm{kg} / \mathrm{min}$. The patient maintained stable vital signs and consciousness until the end of the operation. The total operation time was 80 minutes, in addition, $700 \mathrm{ml}$ of Hartmann solution and $500 \mathrm{ml}$ of hydroxyethyl starch (Voluven ${ }^{\circledR}$, Fresenius Kabi, Germany) were infused. Estimated blood loss was about $500 \mathrm{ml}$ and a urine output was minimal.

After the operation, she was transferred to the surgical intensive care unit (SICU) for an intensive cardiovascular monitoring. The postoperative arterial blood gas analysis, complete blood count, electrolyte, and coagulation tests were normal and only serum Troponin I was mildly elevated $(0.298 \mathrm{ng} / \mathrm{ml})$. Also, the ECG and chest image showed no abnormality. Dopamine infusion was stopped, and she showed stable vital signs. After 27 hours of close observation in the SICU, she was transferred to the general ward. Postoperative cardiovascular evaluation found no abnormality, and the cardiologist recommended conservative management. After 4 days in the general ward, she was discharged without any complications. The perioperative and intraoperative vital signs are summarized in Table 1.

\section{Discussion}

In this case, the possible cause of cardiac arrest includes: vasovagal response; ventricular arrhythmia; amniotic fluid embolism (AFE); and anaphylaxis after the administration of Cefazolin. We believe that the vasovagal response should be the most probable cause of her cardiac arrest.

Firstly, although the history of NSVT, of course, was one issue for abnormal cardiac activity, it was not the direct cause of cardiac arrest. Her intra- and post-operative ECG showed no ventricular arrhythmia. In addition, the progressively slowing HR, immediately before the cardiac arrest, suggests their supraventricular origin. Secondly, unique characteristics of AFE, such as disseminated intravascular coagulation, pulmonary edema, and heart failure, were not observed. Her postoperative coagulation test, chest radiography, and echocardiography were all within normal limits. Also, the patient had a good response

Table. 1. Perioperative and Intraoperative Vital Signs

\begin{tabular}{|c|c|c|c|c|c|c|c|c|c|c|c|c|}
\hline & Ward & OR & S/A & HypoTN & Delivery & Arrest & $\sim 1 \min$ & $\sim 2 \min$ & $\sim 3 \min$ & $\sim 4 \min$ & $\sim 5 \min$ & SICU \\
\hline HR (bpm) & 95 & 94 & 92 & 96 & 98 & Asystole (CPR) & $>100(\mathrm{CPR})$ & $>100(\mathrm{CPR})$ & 83 (ROSC) & 92 & 90 & 90 \\
\hline SBP (mmHg) & 110 & 132 & 102 & 88 & 128 & 48 & 53 & 60 & 85 & 104 & 104 & 120 \\
\hline DBP (mmHg) & 63 & 70 & 63 & 40 & 68 & 29 & 38 & 47 & 69 & 67 & 73 & 49 \\
\hline $\mathrm{SpO}_{2}(\%)$ & N/A & 100 & 100 & 100 & 100 & N/A & $\mathrm{N} / \mathrm{A}$ & $\mathrm{N} / \mathrm{A}$ & 100 & 100 & 100 & 100 \\
\hline
\end{tabular}

HR: heart rate, SBP: systolic blood pressure, DBP: diastolic blood pressure, OR: operating room, S/A: at the induction of spinal anesthesia, HypoTN: hypotension after the induction of spinal anesthesia, $\mathrm{SpO}_{2}$ : oxygen saturation by pulse oximetry, SICU: surgical intensive care unit, CPR: cardiopulmonary resuscitation, ROSC: return of spontaneous circulation, N/A: not available.

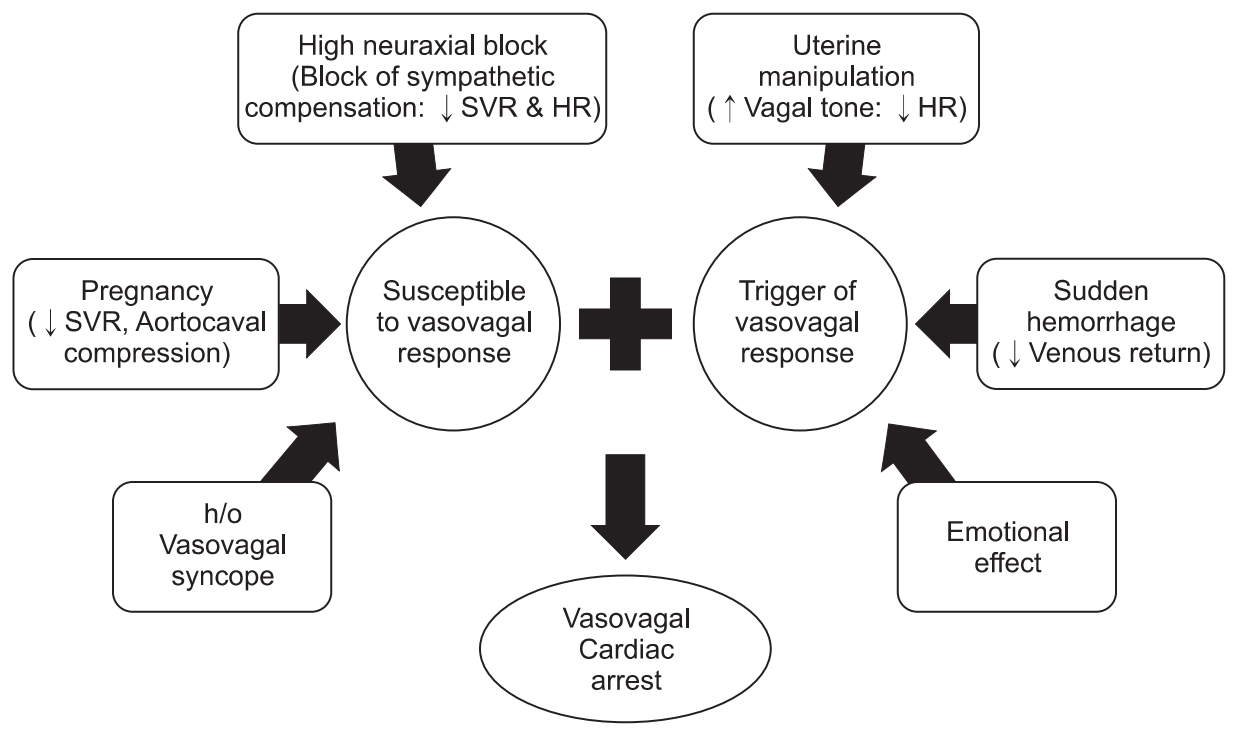

Fig. 1. Risk factors of vasovagal cardiac arrest in this case. HR: heart rate, SVR: systemic vascular resistance, h/o: History of. 
to CPR and recovered without complication, while cardiac arrest originated from AFE usually has a poor response to CPR, and thus, exhibits a high mortality rate. Thirdly, intravenous administration of Cefazolin can cause severe anaphylaxis. Anaphylaxis is a severe systemic allergic reaction, and is typically presented with multi-organ system involvement. Nausea is a common gastrointestinal symptom of anaphylaxis, and acute anaphylactic shock can trigger Bezold-Jarisch reflex. However, her preoperative allergy skin test (AST) result was negative for Cefazolin, and there was no other evidence of anaphylaxis; itchiness, flushing, or swelling of the skin and mucosa, as well as wheezing or stridor in the respiratory system. On the other hand, many factors in this case support vasovagal response, as the cause of sudden cardiac arrest (Fig. 1). The patient was a full term woman, and had several vasovagal episodes before the surgery; she was more susceptible for vasovagal response. Term pregnant women experience aortocaval compression in the supine position, followed by a decrease in the right atrial filling pressure. In about $8 \%$ of the pregnant women, cardiovascular system cannot compensate for severe abrupt drops in venous return, and hence, the supine hypotensive syndrome develops. Furthermore, term pregnant women present significant decrease in systemic vascular resistance $(-25 \%)$ by the development of a low resistance vascular bed in the placenta and vasodilation caused by prostacyclin, estrogens, and progesterone. Not only an increase in the plasma volume $(+50 \%)$, but also a compensatory sympathetic activation with increased HR $(+25 \%)$, stroke volume $(+25 \%)$, and cardiac output $(+50 \%)$ maintain hemodynamic stability. Usually, sympathetic activity reaches a peak at the term [12]. However, this compensatory sympathetic activity is blocked during neuraxial anesthesia. In Cesarean section, due to a lower maternal mortality and complication rate, neuraxial anesthesia is preferred to a general anesthesia. A mid- or high-thoracic level block (T4) is necessary to comfort the patient. Therefore, the incidence of hypotension (63\%) and severe bradycardia (6.7\%) are much higher [13]. Introduction of the left lateral tilting position, and a massive pre-hydration before neuraxial anesthesia (10-20 ml/ $\mathrm{kg}$ of crystalloid solution or $250-500 \mathrm{ml}$ of colloid solution) have reduced the incidence of hypotension and cardiac arrest during a Cesarean section. However there are still some reports of vasovagal cardiac arrest, despite these precautions $[10,11]$.

The patient's block level was T3; in this block level, the sympathetic vasomotor tone, arising from T5 to L1, is completely blocked, while the sympathetic cardiac accelerator fibers that are arising from $\mathrm{T} 1-\mathrm{T} 4$ are only partially blocked. Her baseline HR, before the neuraxial block, was 90-100 bpm. Further, no significant changes were observed after the induction of spinal anesthesia. When the patient showed nausea and mild hypotension after a spinal anesthesia, judging from the HR, we administrated crystalloid and phenylephrine. It was effective, and she showed stable vital signs, until the beginning of placental expulsion. However, similar to that of the previous reports, this case showed an abrupt onset of severe sinus bradycardia, and cardiac arrest in a patient who was hemodynamically stable, well oxygenated, and comfortable $[6,10,11]$.

We suggest an inversion and traction of the uterus, during the placental expulsion, as the triggers of catastrophic vasovagal response. A transient uterine inversion occurred during the placental expulsion, and immediately after that, cardiac arrest occurred. There are some case reports of hemodynamic instability, during the placental expulsion, due to an inversion or traction of the uterus $[14,15]$. During a uterine manipulation, stretching of the peritoneal and broad ligaments around the uterus can result in a secondary stretching of infundibulopelvic ligaments; which then can cause vasovagal reflex via the afferent nerve stimulation. In these cases, patient's hemodynamic status improved dramatically, immediately after the reposition of the uterus, which suggests a vasovagal response.

Our patient's cardiovascular system could not compensate for this vasovagal response, potentiated by a high neuraxial anesthesia with impaired reflex tachycardia and vasoconstriction in blocked segment; eventually leading to a cardiac arrest. Acute hemorrhage, during placental expulsion, may have played a role in vasovagal response, which decreased the venous return. However, it does not seem to be the main cause of vasovagal cardiac arrest. The absolute blood loss during a placental expulsion was not severe. Paradoxically, due to low perfusion pressure during CPR, blood loss was minimal. The total estimated blood loss of this case was about $500 \mathrm{ml}$; which is less than the average blood loss during a Cesarean section.

Anxiety and fear make the management of a patient difficult, and can cause vasovagal response. However, an appropriate sedation may reduce such risk. On the other hand, due to the risk of maternal desaturation, neonatal sedation and respiratory problem, our hospital provides intraoperative sedation, after the confirmation of an uneventful childbirth and placental expulsion. For patients with severe anxiety and fear, we provide general anesthesia. Alert patients can give us important information by complaining their symptoms that cannot be obtained by monitoring vital signs. Also, alert patients can experience and remember the moment of childbirth and the first contact between mother and baby. In this case, the patient did not let out her feelings through the operation; she did not seem to be anxious, nervous or restless. However, Cesarean section in an unfamiliar environment would have put an extremely heavy burden on the patient, and childbirth may cause huge emotional change. After childbirth, she smiled at 
her baby being examined by the nurses, and consequently, began to tear. Anxiety and emotional change may have played an important role in triggering or exaggerating a vasovagal response.

Aggressive treatments, during CPR, including intravenous administration of epinephrine, rapid hydration and Trendelenburg position, were effective in this case, and no postoperative complications were observed. Similar rapid recovery and relatively satisfactory outcomes were reported in the previous cases of vasovagal cardiac arrest $[4,6,10,11]$. Their treatment included early recognition, chest compression, manual ventilation with $100 \%$ oxygen, rapid hydration, and administration of atropine, ephedrine, phenylephrine and epinephrine.

How could this patient have been better managed? Firstly, a careful history taking regarding fainting or syncope, and proper preoperative evaluations, such as head-up tilt test would have reduced the risk of unprepared vasovagal reaction. Secondly, a careful and slow induction of epidural anesthesia would have reduced the risk of high neuraxial anesthesia. Thirdly, maintenance of adequate preload with sufficient hydration, before neuraxial anesthesia, and placental expulsion would have prevented sudden decrease in the venous return. Fourthly, intravenous administration of ephedrine, rather than phenylephrine, after neuraxial anesthesia induced hypotension would have prevented severe reflex bradycardia, by stimulating both alpha- and beta-adrenergic receptor systems. Finally, appropriate intraoperative sedation would have reduced the anxiety of the patient, and possibility of a vasovagal response.

Vasovagal response is just a reflex, making it difficult to predict; hence, hard to prevent. The hemodynamic collapse during a severe vasovagal response takes place in an instant; in the present case, asystole occurred in less than 5-10 seconds. Without constant and careful attention, and appropriate preparation, early recognition and treatment are unlikely to be achieved. Fortunately, we continuously monitored the patient's invasive arterial pressure in preparation for ventricular arrhythmias, so that immediate assessment and treatment were possible. However, monitoring of an invasive arterial pressure is not indicated for routine Cesarean section, and pain and/or anxiety, during arterial cannulation, can trigger the vasovagal response. Thus, the risks and benefits of invasive pressure monitoring should be considered, carefully, before making any clinical decision, based on the individual patient's condition and characteristics of previous vasovagal episodes.

In conclusion, we described a patient with severe vasovagal cardiac arrest, during the spinal anesthesia for Cesarean section. In Korea, this is the first case report of vasovagal cardiac arrest, during a spinal anesthesia for Cesarean section.
This case illustrates a serious potential risk of vasovagal response superimposed on neuraxial anesthesia, during a Cesarean section. Vasovagal response should be suspected, among the many potential causes of cardiac arrest during a Cesarean section, especially when cardiac arrest occurs during placental expulsion. Careful history taking and preoperative evaluation, appropriate preload management, early recognition of vasovagal response by vigilant monitoring, and prompt treatment of hemodynamic collapse, were important lessons we have learned in this case.

\section{References}

1. Kinsella SM, Tuckey JP. Perioperative bradycardia and asystole: relationship to vasovagal syncope and the Bezold-Jarisch reflex. Br J Anaesth 2001; 86: 859-68.

2. Hart PS, Yanny W. Needle phobia and malignant vasovagal syndrome. Anaesthesia 1998; 53: 1002-4.

3. McConachie I. Vasovagal asystole during spinal anaesthesia. Anaesthesia 1991; 46: 281-2.

4. Sprung J, Abdelmalak B, Schoenwald PK. Vasovagal cardiac arrest during the insertion of an epidural catheter and before the administration of epidural medication. Anesth Analg 1998; 86: 1263-5.

5. Pollard JB. Cardiac arrest during spinal anesthesia: common mechanisms and strategies for prevention. Anesth Analg 2001; 92: 252-6.

6. Geffin B, Shapiro L. Sinus bradycardia and asystole during spinal and epidural anesthesia: a report of 13 cases. J Clin Anesth 1998; 10: 278-85.

7. Huang MH, Roeske WR, Hu H, Indik JH, Marcus FI. Postural position and neurocardiogenic syncope in late pregnancy. Am J Cardiol 2003; 92: 1252-3.

8. Doyle DJ, Mark PW. Reflex bradycardia during surgery. Can J Anaesth 1990; 37: 219-22.

9. Coventry DM, McMenemin I, Lawrie S. Bradycardia during intraabdominal surgery. Modification by pre-operative anticholinergic agents. Anaesthesia 1987; 42: 835-9.

10. Watkins EJ, Dresner M, Calow CE. Severe vasovagal attack during regional anaesthesia for caesarean section. Br J Anaesth 2000; 84: 118-20.

11. Scull TJ, Carli F. Cardiac arrest after Caesarean section under subarachnoid block. Br J Anaesth 1996; 77: 274-6.

12. Chang AB. Physiologic changes of pregnancy. In: Obstetric Anesthesia: Principles and Practice. 3rd ed. Edited by Chestnut DH: Philadelphia, Elsevier Mosby. 2004, pp 18-21.

13. Shen CL, Ho YY, Hung YC, Chen PL. Arrhythmias during spinal anesthesia for Cesarean section. Can J Anaesth 2000; 47: 393-7.

14. Emmott RS, Bennett A. Acute inversion of the uterus at caesarean section. Implications for the anaesthetist. Anaesthesia 1988; 43: 118-20.

15. Rho JY, Park KS, Park SY, Do SH. Replacement of uterine inversion by the induction of general anesthesia -A case report-. Korean J Anesthesiol 2004; 47: 284-6. 